\title{
A Novel Algorithm for Breast Mass Classification in Digital Mammography Based on Feature Fusion
}

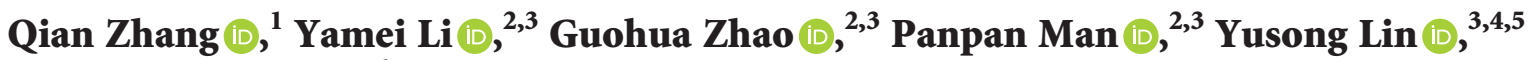 \\ and Meiyun Wang $\mathbb{D}^{6}$ \\ ${ }^{1}$ School of Computer Science, Zhongyuan University of Technology, Zhengzhou 450007, China \\ ${ }^{2}$ School of Information Engineering, Zhengzhou University, Zhengzhou 450001, China \\ ${ }^{3}$ Collaborative Innovation Center for Internet Healthcare, Zhengzhou University, Zhengzhou 450052, China \\ ${ }^{4}$ School of Software, Zhengzhou University, Zhengzhou 450002, China \\ ${ }^{5}$ Hanwei IoT Institute, Zhengzhou University, Zhengzhou 450002, China \\ ${ }^{6}$ Department of Radiology, People's Hospital of Zhengzhou University, Zhengzhou 450003, China
}

Correspondence should be addressed to Meiyun Wang; mywang@ha.edu.cn

Received 30 April 2020; Revised 7 December 2020; Accepted 13 December 2020; Published 22 December 2020

Academic Editor: Aiping Liu

Copyright (c) 2020 Qian Zhang et al. This is an open access article distributed under the Creative Commons Attribution License, which permits unrestricted use, distribution, and reproduction in any medium, provided the original work is properly cited.

\begin{abstract}
Prompt diagnosis of benign and malignant breast masses is essential for early breast cancer screening. Convolutional neural networks (CNNs) can be used to assist in the classification of benign and malignant breast masses. A persistent problem in current mammography mass classification via $\mathrm{CNN}$ is the lack of local-invariant features, which cannot effectively respond to geometric image transformations or changes caused by imaging angles. In this study, a novel model that trains both texton representation and deep CNN representation for mass classification tasks is proposed. Rotation-invariant features provided by the maximum response filter bank are incorporated with the $\mathrm{CNN}$-based classification. The fusion after implementing the reduction approach is used to address the deficiencies of CNN in extracting mass features. This model is tested on public datasets, CBIS-DDSM, and a combined dataset, namely, mini-MIAS and INbreast. The fusion after implementing the reduction approach on the CBIS-DDSM dataset outperforms that of the other models in terms of area under the receiver operating curve (0.97), accuracy (94.30\%), and specificity (97.19\%). Therefore, our proposed method can be integrated with computer-aided diagnosis systems to achieve precise screening of breast masses.
\end{abstract}

\section{Introduction}

Breast cancer ranks first in morbidity and mortality amongst all diseases that affect females [1]. Recent research from the International Agency for Research on Cancer indicates that the incidence of breast cancer in China is gradually increasing, with more than 300,000 women diagnosed with breast cancer every year [2]. Mammography is widely used in the early screening of breast cancer because of its high diagnostic sensitivity for small lesions [3].

For radiologists, the detection or interpretation of breast masses via digital mammography is a time-consuming task [4]. Computer-aided diagnosis (CAD) systems are utilized in breast cancer diagnosis to reduce the burden of reading digital mammography images on radiologists and improve their diagnostic efficiency. Amongst various breast abnormalities (e.g., masses, microcalcifications, architectural distortions, and asymmetry), breast masses are difficult to distinguish from similar backgrounds because of their variable size and low contrast, both of which affect the diagnostic results.

Images obtained from X-rays display various body postures or different imaging angles, and effectively identifying the texture to be measured at different angles is important when performing texture analysis on the mass [5]. Convolutional neural networks (CNNs) can directly extract objective features from images without relying on feature extraction and manual selection [6]. A persistent problem in 
current mammography mass classification via $\mathrm{CNN}$ is the lack of local-invariant features, which cannot effectively respond to geometric image transformations or changes caused by imaging angles. This challenge can only be alleviated by manually manipulating the image rotation to augment the dataset, which is not effective for fine rotation.

Image texture is defined as a function of the spatial variation in pixel intensity (grey value) [7]. Texture analysis can systematically characterize complex visual patterns. The maximum response (MR) filter bank used in our study can deal with the rotation invariance of local images [8]. Moreover, the MR filter bank can effectively capture slight changes in the texture of images. Learned representations based on the MR filter bank can precisely model multiscale and multidirectional information that is important for breast mass diagnosis. However, a single texture feature cannot describe deep image features.

In summary, the feature representations of these two approaches are integrated into a single model. A novel method harnessing the complementary ability of fused rotation-invariant filters and deep learning for breast mass classification is proposed in this work. The MR filter bank is convolved with the images to generate textons, which are then fused with the feature representation extracted by ImageNet pre-trained CNN. The discriminative ability of the rotation-invariant filter banks and deep learning features in classifying benign and malignant masses is tested. Direct fusion and fusion after reduction approaches are implemented to compare and select the best classification model for breast mass diagnosis.

The proposed method has the following advantages:

(1) Given that body postures or imaging angles vary in mammography mass images, a rotation-invariant filter set is used to analyze the texture of mass images

(2) This study is the first to harness the complementary discriminative power of rotation-invariant and deep learning representations for breast mass classification

(3) The fusion after implementing the reduction approach can harness better the complementarity between two groups of features and markedly improve the performance of breast mass classification

\section{Related Work}

Texture analysis can systematically characterize complex visual patterns. Via this approach, suspected regions can be examined by analyzing texture features. Haralick et al. [9] proposed the method of grey level co-occurrence matrices (GLCM), which is extensively used in image recognition and classification. Da Rocha et al. [10] combined diversity indices with GLCM as a way of describing the texture of breast tissues. Through this combination, they obtained an accuracy of $88.31 \%$. Abdalla et al. [11] adopted the GLCM to extract the texture features of images from the Digital Database for Screening Mammography (DDSM) and achieved an accuracy of $91.67 \%$. Co-occurrence matrices are also the main tools for texture analysis. By combining two- dimensional discrete wavelet transform with matrices to extract features from mammographic images, Beura et al. [12] achieved an accuracy of $97.4 \%$. Texton is an effective tool for texture analysis. It is usually obtained through a filter set-based feature extraction approach that characterizes various pixel relationships in a specific area of an image [13-15]. Acharya et al. [16] applied the MR filter bank to convolve with images to generate textons. This approach attained $96 \%$ accuracy, demonstrating the effectiveness of the textons generated by the MR filter bank for classifying breast datasets. However, this approach does not consider deeper image features. A single texture feature cannot fully describe deep image features. Furthermore, the settings of the initial parameters of these traditional methods heavily rely on experience.

With the rapid development of deep learning, convolutional neural networks (CNNs) can directly extract objective features from images without relying on feature extraction and manual selection [17]. A single deep learning model is effective in fields involved in disease diagnosis, such as radiology and ophthalmology [18]. A previous study reported that deep learning outperforms physicians in classifying benign and malignant breast lesions [19]. Carneiro et al. [20] showed that pre-trained deep learning models can be applied to medical imaging. An area under the curve (AUC) of 0.90 was achieved in various mammogram datasets (e.g., INbreast and DDSM). Qiu et al. [21] recognized features from breast images through $\mathrm{CNN}$ and $\max$ pooling concepts. A prior study implemented a CNN along with intensity information and a decision mechanism to classify breast masses [22]. The increasing availability of large medical datasets facilitates the satisfactory performance of CNNs in assisting breast cancer diagnosis [23-25]. However, CNNs cannot explicitly realize rotation invariance of local images and thus cannot effectively respond to geometric image transformations or changes caused by imaging angles.

Some researchers sought to develop a methodology that combines texture analysis and deep learning for feature extraction. Wang et al. [26] explored a breast CAD method based on feature fusion with $\mathrm{CNN}$ deep features, texture features, and density features. He et al. [27] established a classification model on the basis of extracted textures and deep CNN features for evaluating diagnostic performance on differentiating malignant masses. They proved that the deep learning classification model for breast lesions, which was established according to image texture characteristics, can effectively differentiate malignant masses. These fusion methods are merely simple extensions at the feature level, and they do not consider the characteristics of mammography mass images.

\section{Materials and Methods}

The framework of the proposed method in this work is shown in Figure 1. The MR filter bank is convolved with the images to generate textons, which are then fused with the feature representation extracted by ImageNet pre-trained $\mathrm{CNN}$. Direct fusion and fusion after reduction approaches 


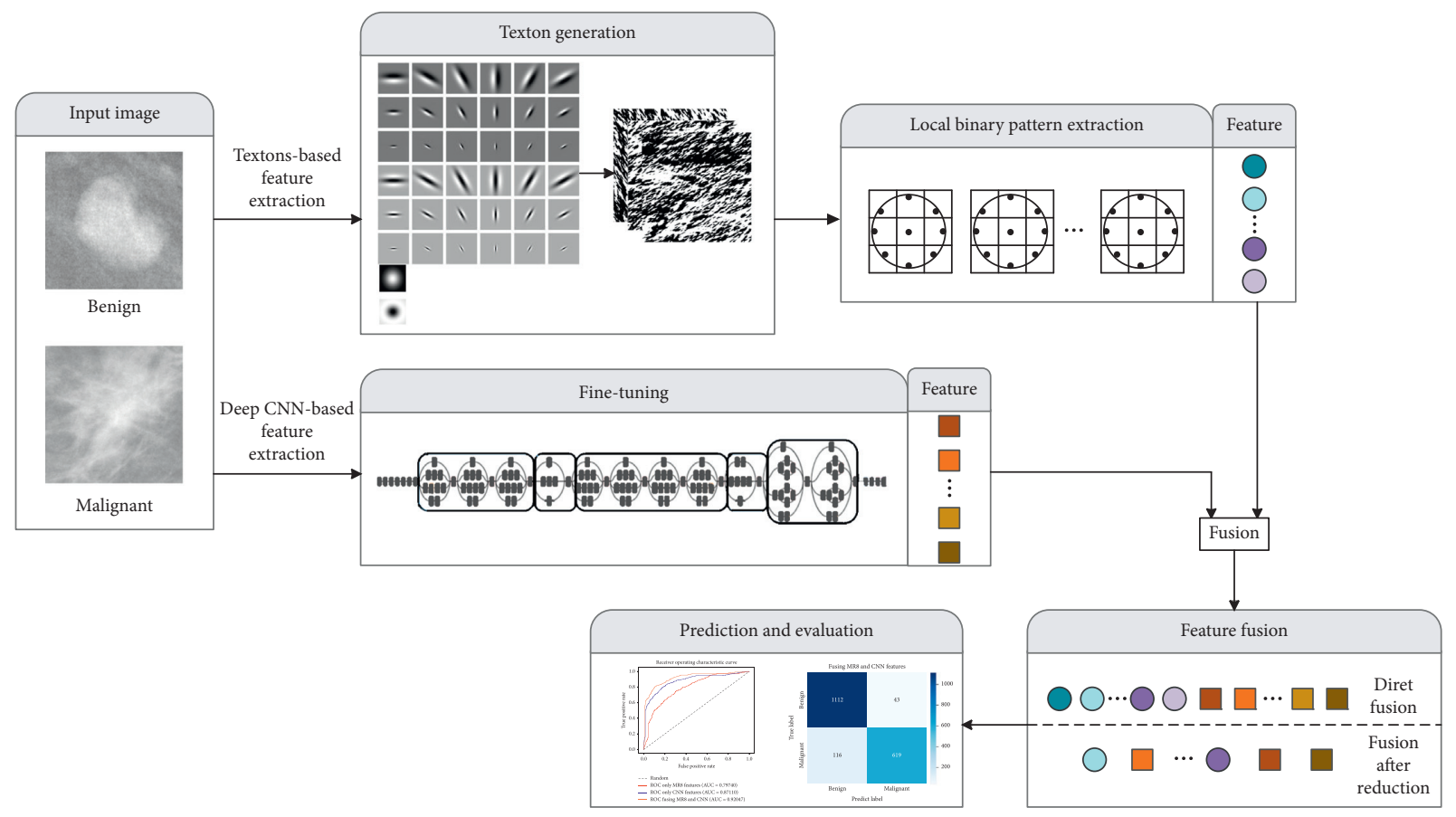

FIgURe 1: Proposed method framework for mammogram mass classification.

are implemented to compare and select the best classification model for breast mass diagnosis.

3.1. Learning Representation from Texton. Texton can be generated using a filter set-based feature extraction approach that characterizes various pixel relationships in a specific area of an image. In past decades, many effective filter banks, such as Leung-Malik [28], Schmid [15], and MR filters [8], have been used to generate textons. The current study uses the MR filter bank containing isotropic and anisotropic filters to produce a satisfactory response to directional textures. Several studies have indicated that the MR filter bank can obtain textons with powerful discrimination $[7,29]$.

3.1.1. MR Filter Bank. The MR filter bank consists of 38 filters. As shown in Figure 2, six orientations at three scales exist for two oriented filters in the first and second derivatives, thereby forming 36 anisotropic filters. The two isotropic filters are the Gaussian and Laplacian of Gaussian (LOG) filters.

If $G$ is a Gaussian kernel function, then the first and second derivatives Gaussian filter can be defined as

$$
\begin{aligned}
G^{\prime} & =G_{x} \cos \theta+G_{y} \sin \theta . \\
G^{\prime \prime} & =G_{x x} \cos ^{2} \theta+G_{y y} \sin ^{2} \theta-2 G_{x y} \cos \theta \sin \theta .
\end{aligned}
$$

LOG can be defined as

$$
\text { LOG }=\nabla^{2} G=G_{x x}+G_{y y} .
$$

The MR8 filter bank is used to achieve rotational invariance. It yields eight responses: six responses from the three scales for two filters and two responses from the

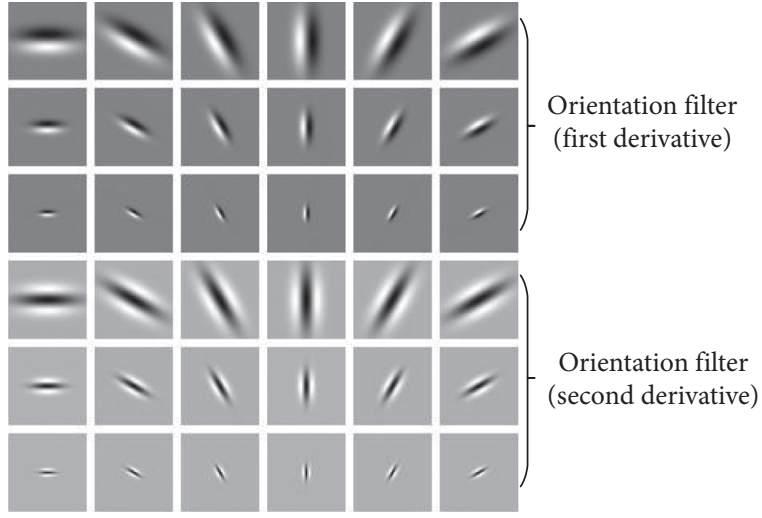

. J Gaussian filter
e J Laplacian of gaussian filter

Figure 2: MR filter bank.

Gaussian and LOG filters. Using the MR8 filter bank to convolve with the images reduces the 38 filter responses to 8 . This step not only reduces the dimensionality of the responses but also implies rotation invariance. Compared with the traditional rotation invariance filter, the MR8 filter bank can calculate the statistical information of high-order symbiosis in the relevant direction. Such information can help distinguish textures that are visually similar to the mass in its surrounding area.

3.1.2. Local Binary Pattern Extraction. After the MR8 filter bank is used to generate textons, the local binary pattern (LBP) is then employed to extract features. LBP is a simple 
method that can effectively describe local image features by quantifying differences between the grey value of the neighborhood and the central pixel [30]. The texture descriptor is rotation-invariant and is not affected by brightness fluctuations during recognition, which can avoid the variation caused by different angles or imaging times of the mammography images. LBP can be defined as

$$
\operatorname{LBP}_{P, R}\left(x_{c}, y_{c}\right)= \begin{cases}\sum_{n=0}^{P-1} s\left(g_{n}-g_{c}\right), & U\left(\operatorname{LBP}_{P, R}\right) \leq 2, \\ P+1, & \text { otherwise }\end{cases}
$$

where

$$
s(x)= \begin{cases}1, & x \geq 0, \\ 0, & x<0,\end{cases}
$$

$P$ is the number of equally spaced points on the circumference with radius $R, g_{c}$ is the pixel intensity at the centre point, and $U\left(\mathrm{LBP}_{P, R}\right)$ is a measure of uniformity applied to calculate the number of $0-1$ transformations (i.e., from 0 to 1 or vice versa). The working principle of LBP is illustrated in Figure 3. The pixel values on the circumference are compared with the central pixel value to generate a binary value of " 0 " or " 1 " to extract the local contrast information.

The MR8 filter bank is used for the convolution with the images; each filter generates eight filter responses. The LBP algorithm is then applied to extract 36-dimensional feature vectors from each filter response to obtain a texton-based feature representation. A total of $288(36 \times 8)$ dimensional features are extracted from each image.

3.2. Learning Representation from Deep CNN. Given that large-scale training for medical tasks cannot be performed because of the lack of a medical dataset, a pre-trained network is introduced in this study. The InceptionV3 network is applied to the deep feature extraction. The computational cost and memory requirements of this network are lower than those of Residual Neural Network 50, Visual Geometry Group Network, and other networks. The main feature of the inception architecture is the calculation of nonlinear weighted sum modules $(\sigma(W x))$ in each layer, which can be defined as

$$
\sigma\left(\sum_{j=1}^{M} w_{j} x_{j}+b\right)
$$

where $M$ is the number of neurons in this layer, $w_{j} \in W, W$ is the weight matrix, $x$ is the input vector, $b$ is the deviation term, and $\sigma(\cdot)$ is the activation function. The module uses factorization to decompose $5 \times 5$ convolutions into two $1 \mathrm{D}$ $(1 \times 5$ and $5 \times 1)$ and compress the input or the dimension of the output of the previous layer, thereby effectively reducing the complexity and computational cost of the model. The experiment verifies that the effect of using random initialization to retrain the weights in the network is not as good as that using the ImageNet pre-trained network. Hence, the ImageNet pre-trained InceptionV3 model is used as the

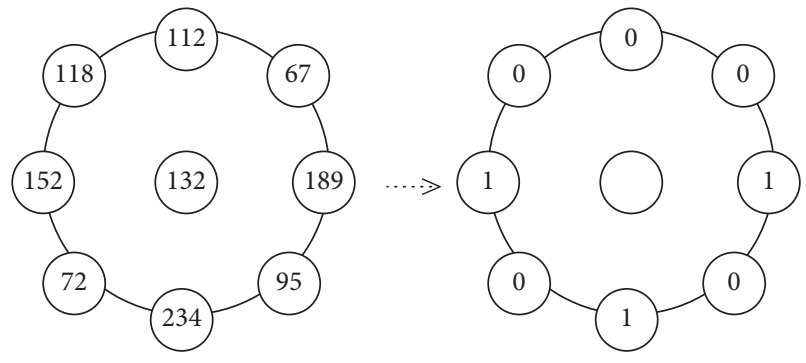

Figure 3: LBP.

feature extractor. To use the pre-trained weights for finetuning, we repeat the grey value matrix of the image in three different channels of RGB to match the input of the retraining architecture. We utilize cross-entropy as the objective function and set the learning rate to be less than the initial learning rate pre-trained by the ImageNet. This step ensures that the network will not completely forget the features learned from the original dataset. The last softmax layer of the network is removed and the 1024-dimensional feature vector extracted from the layer with the largest receptive field (i.e., previous layer of the classification layer, that is, pool5) is selected as the final output features. This layer includes all different learning modes in the previous layer and can obtain the features with a strong discriminatory ability for classification [17].

3.3. Fusing Texton and Deep CNN Features. The idea of feature fusion comes from the early information fusion field, which is used for multisensor fusion in military applications [31]. Feature fusion methods are widely used in image recognition to achieve feature complementation and address the shortcomings of a single feature vector [32]. Two fusion strategies are designed to determine how the complementary information of the two features can be utilized.

3.3.1. Direct Fusion. The most direct way to fuse two sets of feature vectors is to use cascade fusion [33], which can be defined as

$$
X_{F}=\left[X_{\mathrm{MR}}, X_{\mathrm{CNN}}\right],
$$

where $X_{\mathrm{MR}}$ and $X_{\mathrm{CNN}}$ are the texton- and deep CNN-based feature vectors, respectively; $X_{F}$ is the fusion feature vector; and $\operatorname{dim}\left(X_{F}\right)=\operatorname{dim}\left(X_{\mathrm{MR}}\right)+\operatorname{dim}\left(X_{\mathrm{CNN}}\right)$. As described in Sections $3.1 \quad$ and $3.2, \quad \operatorname{dim}\left(X_{\mathrm{MR}}\right)=288 \quad$ and $\operatorname{dim}\left(X_{\mathrm{CNN}}\right)=1024$. The dimension of the deep CNN features is more than three times the feature vectors obtained from MR8. The classification may focus on the deep CNN features and ignore the supplementary information in the textons. Therefore, we design a fusion strategy after feature reduction.

3.3.2. Fusion after Reduction. In this strategy, feature selection is performed on two sets of feature vectors before cascade fusion is executed. Random forest is used for feature selection, which can analyze complex interactive features 
and is extremely robust to noisy and redundant data $[34,35]$. On the basis of the feature importance measurement method, which uses the classification accuracy of Out-of-Bag (OOB) [36], feature subsets are selected according to the sequential forward selection (SFS) method.

The feature importance ranking method based on the classification accuracy of the $\mathrm{OOB}$ can be expressed as follows.

If the feature dimension is $N$, then bootstrap is adopted to extract $M$ datasets. $M$ OOB datasets are also generated accordingly.

Step 1. $m=1$ initialized and a decision tree $T_{m}$ is created on the training set.

Step 2. The classification accuracy of the mth OOB dataset $A_{m}^{\text {oob }}$ is calculated.

Step 3. The feature $\left.x_{i}(i=1,2, \cdots, N)\right)$ is disturbed in the OOB dataset, and the accuracy $A_{m, i}^{\text {oob }}$ is recalculated.

Step 4. Steps 2 and 3 are repeated for $m=2,3, \cdots, M$.

Step 5. The importance of $x_{i}$ is calculated using

$$
D_{i}=\frac{1}{M} \sum_{m=1}^{M}\left(A_{m}^{\mathrm{oob}}-A_{m, i}^{\mathrm{oob}}\right) .
$$

Step 6. It is sorted in descending order. A high feature ranking indicates high importance.

Fivefold cross-validation is used to select more effective features. Subsequently, the OOB dataset is utilized to obtain the rank of importance and calculate accuracy. The sorted set of results with the most satisfactory classification effect is then selected, and the optimal feature subset is obtained using the SFS method. Finally, the cascade fusion of the two sets of features is executed.

3.4. Classification. The classifier is used to determine the relationship amongst the sets of attributes to predict the possible attribution results [37]. After the classifier is trained, the test data are fed into the network to predict the category and evaluate the performance of the algorithm. The following classifiers are used to classify benign and malignant masses.

For the direct fusion, the softmax in InceptionV 3 is used as the classifier and the fused feature as its input. A dropout is added to the classification layer to enhance the robustness of the network. The stochastic gradient descent is used to minimize cross-entropy cost function.

For the fusion after reduction, a support vector machine (SVM) is utilized to distinguish benign and malignant masses on the basis of low-dimensional features. SVM is a supervised machine learning method widely used in statistical classification and regression analyses [38]. This technique can identify the best compromise between learning accuracy and learning ability of a specific training sample. In this study, the SVM based on radial basis function (RBF) kernel is used, and the features fused after reduction are used as inputs to obtain the probability of classifying the masses as benign or malignant.

Algorithm 1 shows the workflow of the method proposed here.

\section{Results and Discussion}

4.1. Image Databases and Preprocessing. In our study, we utilized three digital databases for screening mammography images, namely, Curated Breast Imaging Subset of DDSM (CBIS-DDSM) [39], INbreast [40], and Mammographic Image Analysis Society (mini-MIAS) [41] to evaluate performance of the proposed method.

4.1.1. CBIS-DDSM. The CBIS-DDSM dataset is the curated breast imaging subset of DDSM. It consists of 861 mass cases and full mammography images, including mediolateral oblique and craniocaudal views of mammograms (i.e., 912 benign and 784 malignant masses).

4.1.2. INbreast. The INbreast dataset was created by the Breast Research Group, INESCPorto, Portugal. It contains images of 115 patients for a total of 410 images, including images of masses, calcifications, and other abnormalities. It contains a total of 112 masses (i.e., 36 benign and 76 malignant masses).

4.1.3. Mini-MIAS. The mini-MIAS, which is provided by the Mammographic Image Analysis Society, London, UK, dataset contains 322 mammogram images obtained from 161 women. It contains a total of 70 available mass images (i.e., 40 benign and 30 malignant masses).

Given that the sample sizes of INbreast and mini-MIAS datasets are too small, we merge them into one dataset. Therefore, these three databases are divided into two groups for evaluating the proposed method (Table 1). To render the dataset suitable for the pre-trained network and reduce the running cost, we extract $300 \times 300$ patches centered at masses in the three databases to build our dataset. Next, an adaptive histogram equalization [42] is applied to balance the contrast. For CBIS-DDSM, similar to other medical image classification experiments, the affine transformation is used to rotate the images by $0^{\circ}, 90^{\circ}, 180^{\circ}$, and $270^{\circ}$ and reflect them along the horizontal axes to augment the dataset and avoid overfitting. For INbreast and mini-MIAS, each mass patch is augmented by the aforementioned affine transformation, and then these four images are flipped from left to right to generate eight images for each patch as the second dataset. Finally, each dataset is split into training $(60 \%)$, validation $(10 \%)$, and test (30\%) sets.

4.2. Experiment Settings. The MR8 filter bank is operated in MATLAB and convolve with the mass images to generate textons. The InceptionV3 model based on Keras is used to transfer the pre-trained weights from ImageNet to the mass 
(1) Input: mammography mass image $I_{i}, i=1,2, \ldots, N$

(2) Output: diagnosis results matrix $Y \in \mathbb{R}^{N}$

(3) Calculate the deep feature matrix $X_{\mathrm{CNN}} \in \mathbb{R}^{N \times n_{1}}$

(4) Get textons by convoluting MR8 filter bank with the $I_{i}$

(5) Calculate the textons-based feature matrix $X_{\mathrm{MR}} \in \mathbb{R}^{N \times n_{2}}$ through LBP

(6) if use the direct fusion approach then

(7) Fusion feature $X_{F}=\left[X_{\mathrm{MR}}, X_{\mathrm{CNN}}\right], X_{F} \in \mathbb{R}^{N \times\left(n_{1}+n_{2}\right)}$

(8) Train softmax classifier with $X_{F}$ and predict $I_{i}$

(9) Return $Y$

(10) end if

(11) if use the fusion after reduction approach then

(12) Calculate the subset $X_{\mathrm{CNN}}^{\prime} \in \mathbb{R}^{N \times n_{3}}$ of $X_{\mathrm{CNN}}$ by random forest

(13) Calculate the subset $X_{\mathrm{MR}}^{\prime} \in \mathbb{R}^{N \times n_{4}}$ of $X_{\mathrm{MR}}$ by random forest

(14) Fusion feature $X_{F}=\left[X_{\mathrm{MR}}^{\prime}, X_{\mathrm{CNN}}^{\prime}\right], X_{F} \in \mathbb{R}^{N \times\left(n_{3}+n_{4}\right)}$

(15) Train SVM classifier with $X_{F}$ and predict $I_{i}$

(16) Return $Y$

(17) end if

Algorithm 1: Mammography mass benign-malignant classification algorithm.

TABLE 1: Digital mammogram dataset.

\begin{tabular}{ccccc}
\hline & Database & Number of benign images & Number of malignant images & Total number of images \\
\hline 1 & CBIS-DDSM & 912 & 784 & 1696 \\
2 & INbreast & 36 & 76 & 182 \\
& Mini-MIAS & 40 & 30 & 182 \\
\hline
\end{tabular}

dataset. Given that mammography mass images are vastly different from ImageNet images, we propose to fine-tune our models to adjust the features of the last convolutional blocks and make them more data-specific. We utilize stochastic gradient descent to fine-tune the network and set the initial learning rate to $10^{-5}$. We divide the initial learning rate by 10 each time the validation error stops improving. Moreover, to improve the results and avoid overfitting, we perform L2 regularization and dropout. When training the SVM model, we employ the train and validation sets to fine-tune the $\mathrm{C}$ parameter for the SVM classifier. After tuning the models and choosing the best hyperparameters, we train each final model by using a stratified fivefold cross-validation with all the data and evaluate each model's performance.

4.3. Evaluation Metrics. In the diagnostic results of medical images, accuracy (Acc), sensitivity (Sens), and specificity (Spec) are the commonly used objective evaluation metrics. The area under the receiver operating characteristic curve (ROC) (i.e., AUC score) is another important metric used to evaluate the performance of diagnostic results. These evaluation metrics are calculated as follows:

$$
\begin{aligned}
\text { Acc } & =\frac{N_{R}}{N}, \\
\text { Sens } & =\frac{\mathrm{TP}}{\mathrm{TP}+\mathrm{FN}}, \\
\text { Spec } & =\frac{\mathrm{TN}}{\mathrm{FP}+\mathrm{TN}} .
\end{aligned}
$$

In benign and malignant mass classification, if the malignant mass is classified as malignant, then the result will be true positive (TP). The result will become true negative (TN) if the benign mass is classified as benign. Similarly, if the benign mass is classified as malignant, then the result will be false positive (FP), which will become false negative (FN) if the malignant mass is classified as benign.

The $\mathrm{k}$-fold cross-validation [43] method is adopted to evaluate the performance of the proposed method. The evaluation metrics in this study are derived from the fivefold cross-validation method.

\subsection{Results and Analysis}

\subsubsection{Direct Fusion}

(1) MR8 Features Only. First, an MR8 filter bank is built, and the filter responses are collected by convolving them with the images. Second, the LBP algorithm is used to extract the 36dimensional feature vectors from each filter response. Finally, the 288-dimensional feature vectors based on MR8 are obtained and used to train the softmax classifier. Fivefold cross-validation is applied to evaluate the average performance of this classifier in benign and malignant mass classification. As shown in Table 2, the AUC score and accuracy obtained by the MR8 features for classification are 0.79 and $70.21 \%$, respectively.

(2) Deep CNN Features Only. The average accuracy obtained by the InceptionV3 model by using the initial weight is $72.21 \%$. When the ImageNet pre-trained InceptionV3 is 
TABLE 2: Comparison of MR8 features, deep CNN features, and direct fusion with CBIS-DDSM.

\begin{tabular}{lcc}
\hline Methods & AUC & Acc \\
\hline MR8 features only & 0.7974 & 0.7021 \\
Deep CNN features only & 0.8711 & 0.7934 \\
Direct fusion of MR8 and deep CNN features & 0.9204 & 0.8002 \\
\hline
\end{tabular}

used to extract the 1024-dimensional feature vectors and train the softmax classifier described in Section 3.4, the classification results demonstrated improvements. The results are shown in the third row of Table 2, where the AUC score is 0.87 and the accuracy is $79.34 \%$.

(3) Direct Fusion. The two features are directly fused using the cascade fusion method to train the softmax classifier. The classification results in the fourth row of Table 2 indicate that the AUC score is 0.92 and the accuracy is $80.02 \%$.

Although the classification results after direct fusion are slightly better than those after using a single feature, the accuracy is almost the same as that when only deep CNN features are applied. This finding might be attributed to the excessively large feature dimension of the fusion, and the feature dimension of deep $\mathrm{CNN}$ being more than three times the feature obtained from MR8. Therefore, the classifier prefers the information contained in the deep CNN features during the classification, which is why the fusion method after feature reduction is developed.

4.4.2. Fusion after Reduction. Random forest and SFS are used to select the feature subsets from the two groups of features. The OOB dataset and fivefold cross-validation are implemented to obtain the importance ranking and select the best set of features for the classification results, respectively. A total of 47 dimensional features are obtained, where 17 are obtained from the feature representation based on MR8 and 30 are obtained from the deep CNN features. The fused features are then fed into the SVM classifier. To obtain an effective comparison of the classification results of the fused features, we train the same SVM classifier by using the two feature subsets. A comparison of the classification results before and after fusion is shown in Table 3. The AUC score and accuracy of the MR8 feature subset only are 0.89 and $80.42 \%$, respectively, in classifying CBIS-DDSM mass images. By comparison, the AUC score and accuracy of the deep CNN feature subset only are 0.92 and $88.67 \%$, respectively. After implementing the reduction strategy, the fusion reaches an accuracy of $94.30 \%$ and an average AUC of 0.97 , an increase of 0.05 and $14.28 \%$, respectively, compared with those of the direct fusion strategy. This result suggests that training the classifier with the fusion features after reduction can better harness the complementarity of these two sets of features.

Figures 4(a) and 4(b) show the ROC curves of the direct fusion and the fusion after reduction, respectively. The three different color curves in each picture reveal that the area under the yellow ROC curve is the largest, which represents the classification result using the fusion features. The curves also confirm that fusion after reduction can effectively combine the advantages of the two features, and the feature representation based on MR8 can provide supplementary information to facilitate the $\mathrm{CNN}$ in classifying benign and malignant masses.

We also construct the fusion after reduction approach on INbreast and mini-MIAS. The classification results are summarized in Table 3. The AUC and accuracy of training the classifier by using the MR8 feature subset only are 0.88 and $88.47 \%$, respectively, which are slightly higher than those of the classification performance by using CNN features. This result is obtained because these two databases are too small despite the fact that we have already augmented the data. CNNs cannot obtain additional effective features from a limited database. In spite of the limited number of datasets, training the classifier with fusion features still improves the performance of the classifier (AUC is 0.93 and accuracy is 93.59\%). This result suggests that our method can achieve high performance even when sample sets are small and image bases are heterogeneous.

Three other machine learning classifiers are used to verify the classification performance of the fused features after reduction. Figure 5 shows the classification results by using k-nearest neighbor classifier ( $\mathrm{kNN})$, SVM based on linear function kernel (SVM-linear), and extreme gradient boosting (XGBoost). Fusion features improve classification performance under all three classifiers (AUC scores are $0.89,0.93$, and 0.96 , respectively). The three classifiers reflect the superiority of the fusion features after reduction. The confusion matrices using XGBoost as displayed in Figure 6 indicate that the number of misclassified benign and malignant masses after fusion is substantially reduced. Specifically, the number of malignant masses incorrectly classified as benign is reduced by nearly half.

4.4.3. Comparative Analysis. To prove the complementary capabilities of MR8 features for CNNs, we adopt two popular deep learning models, namely, ResNet50 and Efficient-B7, to replace the InceptionV3 model in our method. The structure and depth of these models are suitable for medical image classification tasks with few training samples. MR8 + ResNet50 and MR8 + EfficientNet-B7 represent the use of fusion after reduction approach for fusing both MR8 and deep $\mathrm{CNN}$ features. As shown in Table 4, the fused features improve the performance of ResNet50 (ACC and AUC increased by $5 \%$ and 0.02 , respectively) and Efficient-B7 (ACC and AUC increased by $8.55 \%$ and 0.05 , respectively). Therefore, the features obtained from the MR8 filter can effectively compensate for the shortcomings of CNNs in feature extraction.

Various methods have been devised for classifying benign and malignant masses. The best case achieved by the method proposed herein is further compared with that of some recently developed classification methods (Table 4). The performance of our method is superior to that of traditional textural analyses and other machine learning methods $[44,45]$. The performance of two deep learning 
TABLE 3: Comparison of MR8 features, deep CNN features, and fusion after reduction.

\begin{tabular}{lcrr}
\hline Dataset & Methods & AUC & Acc \\
\hline & MR8 features only & 0.8964 & 0.8042 \\
CBIS-DDSM & Deep CNN features only & 0.9262 & 0.8867 \\
& Fusing MR8 and deep CNN features & $\mathbf{0 . 9 7 9 5}$ & 0.9812 \\
INbreast + mini-MIAS & MR8 features only & 0.9430 \\
& Deep CNN features only & 0.8553 & 0.8847 \\
& Fusing MR8 and deep CNN features & 0.9383 & 0.9359 \\
\hline
\end{tabular}

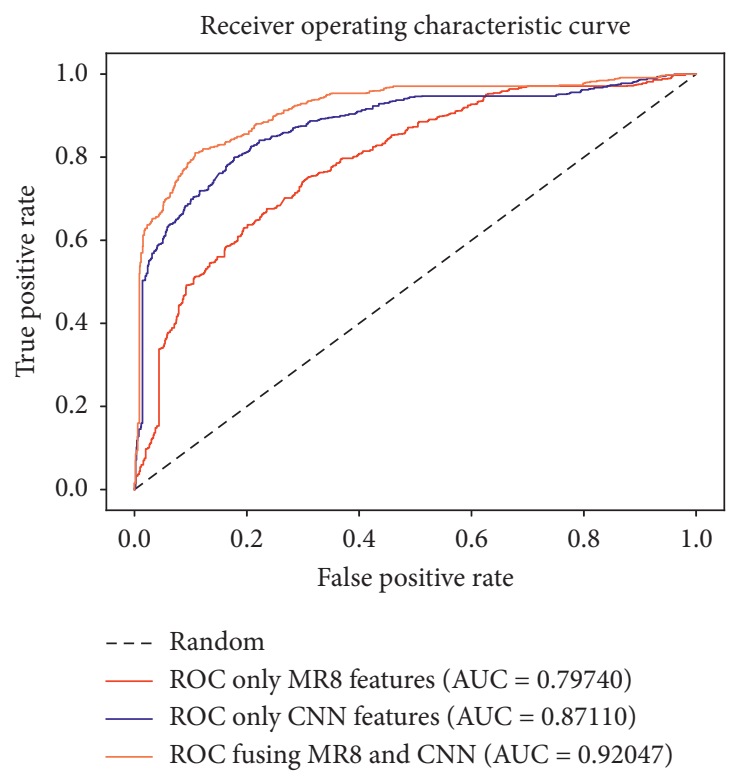

(a)

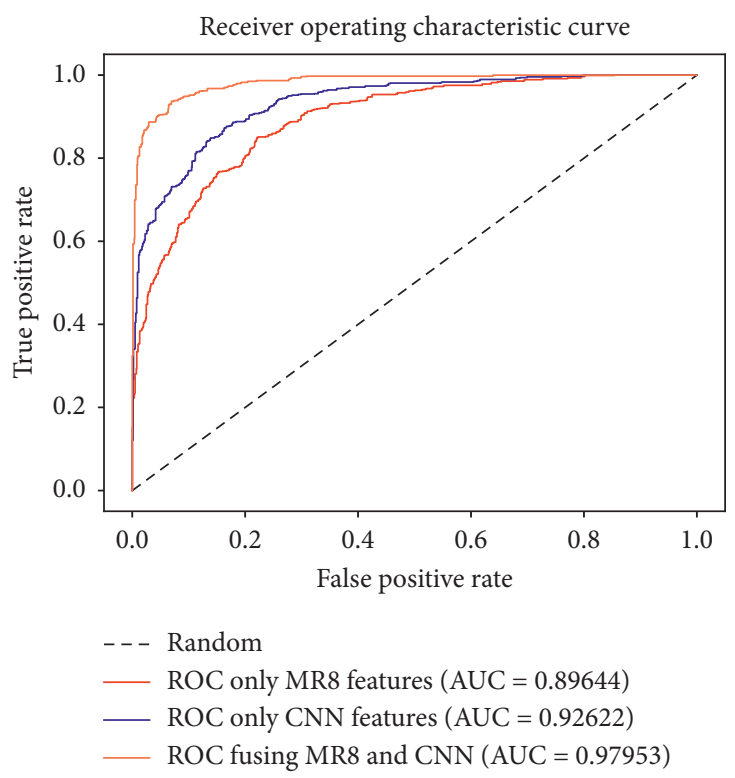

(b)

FIgURE 4: ROC curves of direct fusion and fusion after reduction. (a) The ROC curve of the average performance using direct fusion approach. (b) The ROC curve of the average performance using fusion after reduction approach.

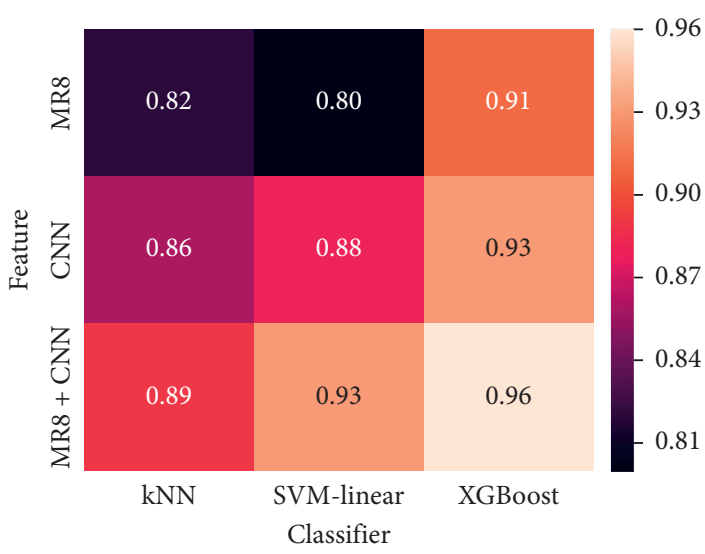

Figure 5: Heat map of AUC score under kNN, SVM-linear, and XG boost using fusion after reduction with CBIS-DDSM.

methods $[46,47]$ is also compared with that of our method. As shown in Table 4, these two methods achieve high sensitivity (98.00\% and $93.83 \%)$. However, their specificity is substantially lower than that of our method, suggesting that they may misclassify more negative masses compared with our method. Overall, the performance of our method is better than that of these two deep learning-based approaches. Moreover, the performance of methods described in $[10,11]$, which integrate multiple features to classify benign and malignant masses, is slightly lower than that of our method. The results establish the superiority and robustness of our proposed method. 


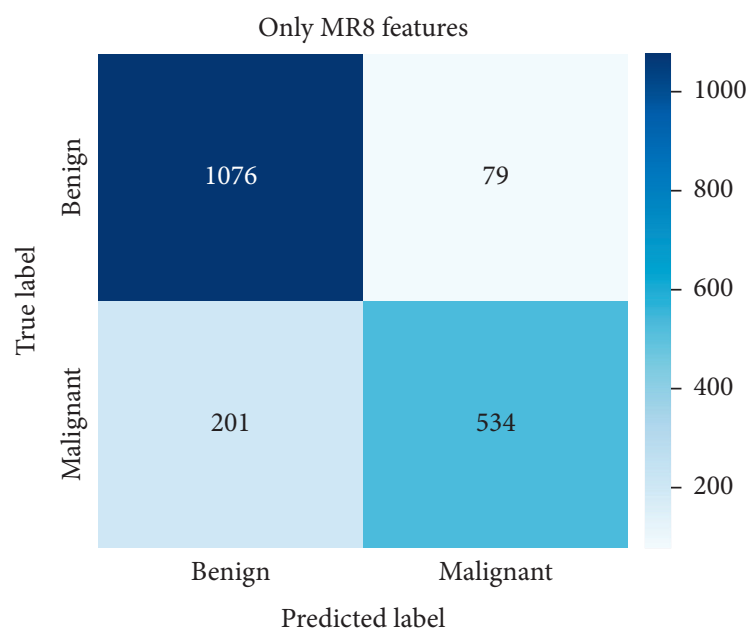

(a)

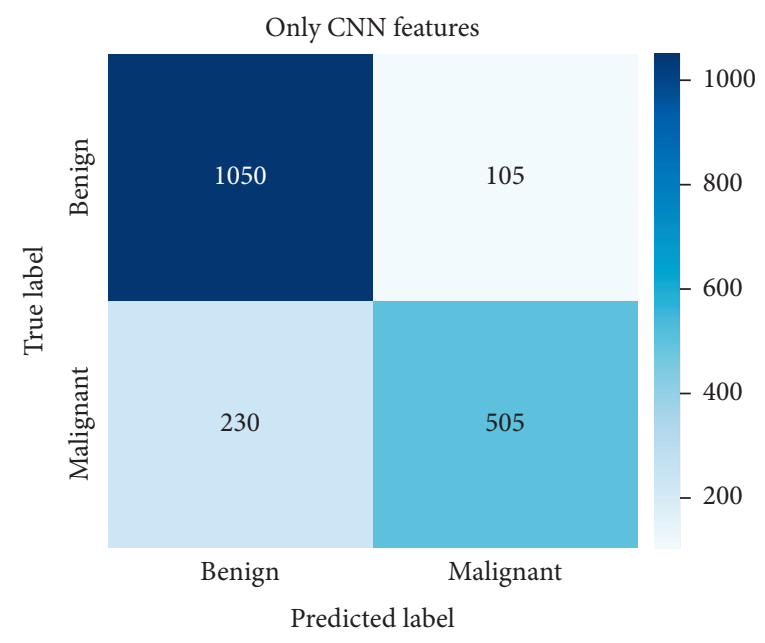

(b)

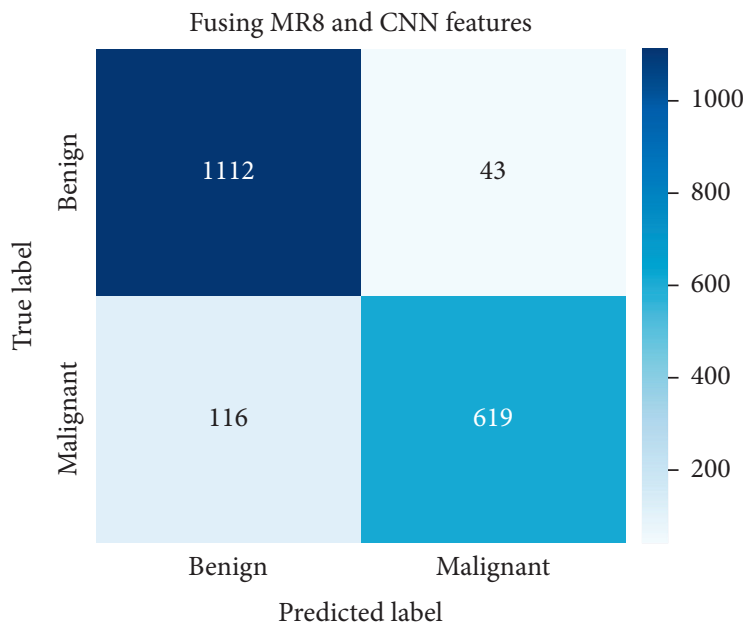

(c)

FIGURE 6: Confusion matrix obtained by XGBoost using fusion after reduction approach with CBIS-DDSM. (a) Only MR8 features. (b) Only deep CNN features. (c) Fusing MR8 and CNN after feature reduction.

TABLE 4: Comparison of proposed method with other mass classification methods.

\begin{tabular}{|c|c|c|c|c|c|}
\hline Dataset & Methods & Sens & Spec & Acc & AUC \\
\hline \multirow{4}{*}{ CBIS-DDSM } & ResNet50 & $77.31 \%$ & $82.07 \%$ & $79.50 \%$ & 0.86 \\
\hline & MR8 + ResNet50 & $83.17 \%$ & $85.94 \%$ & $84.50 \%$ & 0.88 \\
\hline & EfficientNet-B7 & $80.47 \%$ & $81.05 \%$ & $80.75 \%$ & 0.80 \\
\hline & MR8 + EfficientNet-+B7 & $89.88 \%$ & $88.02 \%$ & $89.30 \%$ & 0.85 \\
\hline DDSM & M. Hussain et al. [44] & - & - & $85.53 \%$ & 0.87 \\
\hline BCDR & L. Fangyi et al. [45] & $88.93 \%$ & $93.41 \%$ & $91.65 \%$ & 0.96 \\
\hline INbreast & N. Dhungel [46] & $98.00 \%$ & $70.00 \%$ & $90.00 \%$ & - \\
\hline CBIS-DDSM & C. Yuanqin [47] & $93.83 \%$ & $92.17 \%$ & $93.15 \%$ & 0.95 \\
\hline DDSM & S. V. da rochaa [10] & $85.00 \%$ & $91.89 \%$ & $88.31 \%$ & 0.88 \\
\hline DDSM & Q. Abbas [11] & $92.00 \%$ & $84.20 \%$ & $91.00 \%$ & 0.91 \\
\hline CBIS-DDSM & Proposed method & $89.97 \%$ & $97.91 \%$ & $94.30 \%$ & 0.97 \\
\hline
\end{tabular}

\section{Conclusions}

Body postures or imaging angles vary in mammography masses. Malignant and benign masses may show similar features. Hence, they can be difficult to differentiate. In this study, a novel method based on texton fusion and CNNs for extracting mass features and classifying benign and malignant masses is proposed. Two fusion strategies, namely, 
direct fusion and fusion after reduction, are employed to fuse texton-based feature representations with deep CNN features. Moreover, these fusion strategies are adopted to explore how the complementary discrimination ability of two groups of features can be applied to mass classification tasks. These strategies are tested on the public databases CBIS-DDSM, INbreast, and mini-MIAS. Results show that the fused features can provide useful supplementary information for extracting mass features via CNN. By comparison, the fusion after reduction approach can harness better the complementarity of features extracted from the MR8 filter and deep CNN. Thus, this approach can achieve an accurate classification of benign and malignant masses. Experimental results demonstrate that our method outperforms other state-of-the-art methods without pixel-level annotation. Given that our method does not require any user interaction, it can be easily integrated into CAD systems for breast cancer.

However, mammography images have many pathological classifications, such as microcalcifications and structural distortions. At present, although this method has achieved good results in the classification of benign and malignant masses, it has not been tested in classifing and diagnosing other pathological classifications. With the expansion of our database, we will be able to optimize our method for other pathological classifications of breast images.

\section{Data Availability}

The data used to support the findings of this study are available from the corresponding author upon request.

\section{Conflicts of Interest}

The authors declare that they have no conflicts of interest.

\section{Acknowledgments}

This work was supported by National Natural Science Foundation of China under Grant no. 81772009, Scientific and Technological Research Project of Henan Province under Grant no. 182102310162, and Collaborative Innovation Major Project of Zhengzhou under Grant no. 20XTZX06013.

\section{References}

[1] R. Siegel, C. DeSantis, and A. Jemal, "Colorectal cancer statistics, 2014," CA: A Cancer Journal for Clinicians, vol. 64, no. 2, pp. 104-117, 2014.

[2] J. Ferlay, M. Colombet, I. Soerjomataram et al., "Estimating the global cancer incidence and mortality in 2018: GLOBOCAN sources and methods," International Journal of Cancer, vol. 144, no. 8, pp. 1941-1953, 2019.

[3] C. Lerman, M. Daly, C. Sands et al., "Mammography adherence and psychological distress among women at risk for breast cancer," JNCI Journal of the National Cancer Institute, vol. 85, no. 13, pp. 1074-1080, 1993.

[4] R. A. Smith, K. S. Andrews, D. Brooks et al., "Cancer screening in the United States, 2019: a review of current American Cancer Society guidelines and current issues in cancer screening," Ca-a Cancer Journal for Clinicians, vol. 69, no. 13, pp. 184-210, 2019.

[5] H. D. Cheng, X. J. Shi, R. Min, L. M. Hu, X. P. Cai, and H. N. Du, "Approaches for automated detection and classification of masses in mammograms," Pattern Recognition, vol. 39, no. 4, pp. 646-668, 2006.

[6] A. Esteva, A. Robicquet, B. Ramsundar et al., "A guide to deep learning in healthcare," Nature Medicine, vol. 25, no. 1, pp. 24-29, 2019.

[7] M. Varma and A. Zisserman, "A statistical approach to texture classification from single images," International Journal of Computer Vision, vol. 62, no. 1-2, pp. 61-81, 2005.

[8] J. Geusebroek, A. W. M. Smeulders, and J. Van De Weijer, "Fast anisotropic Gauss filtering," Institute of Electrical and Electronics Engineers Transactions on Image Processing, vol. 12, no. 8, pp. 938-943, 2003.

[9] R. M. Haralick, K. Shanmugam, and I. H. Dinstein, "Textural features for image classification," Institute of Electrical and Electronics Engineers Transactions on Systems, Man, and Cybernetics, vol. 3, no. 6, pp. 610-621, 1973.

[10] S. V. Da Rocha, G. Braz Junior, A. C. Silva, A. C. De Paiva, and M. Gattass, "Texture analysis of masses malignant in mammograms images using a combined approach of diversity index and local binary patterns distribution," Expert Systems with Applications, vol. 66, pp. 7-19, 2016.

[11] Q. Abbas, "Deep CAD: A computer-aided diagnosis system for mammographic masses using deep invariant features," Computers, vol. 5, no. 4, 2016.

[12] S. Beura, B. Majhi, and R. Dash, "Mammogram classification using two dimensional discrete wavelet transform and graylevel co-occurrence matrix for detection of breast cancer," Neurocomputing, vol. 154, pp. 1-14, 2015.

[13] F. Jurie and B. Triggs, "Creating efficient codebooks for visual recognition," in Proceedings of Tenth Ieee International Conference on Computer Vision, pp. 604-610, Institute of Electrical and Electronics Engineers Computer Society, Los Alamitos, CA, USA, October 2005.

[14] U. R. Acharya, H. Fujita, V. K. Sudarshan et al., "An integrated index for identification of fatty liver disease using radon transform and discrete cosine transform features in ultrasound images," Information Fusion, vol. 31, pp. 43-53, 2016.

[15] L. Zhang, X. Ye, T. Lambrou, W. Duan, N. Allinson, and N. J. Dudley, "A supervised texton based approach for automatic segmentation and measurement of the fetal head and femur in 2D ultrasound images," Physics in Medicine and Biology, vol. 61, no. 3, pp. 1095-1115, 2016.

[16] U. R. Acharya, K. M. Meiburger, J. E. Wei Koh et al., "A novel algorithm for breast lesion detection using textons and local configuration pattern features with ultrasound imagery," Institute of Electrical and Electronics Engineers Access, vol. 7, pp. 22829-22842, 2019.

[17] L. Zhou, Z. Zhang, Y.-C. Chen, Z.-Y. Zhao, X.-D. Yin, and H.-B. Jiang, "A deep learning-based radiomics model for differentiating benign and malignant renal tumors," Translational Oncology, vol. 12, no. 2, pp. 292-300, 2019.

[18] D. S. Kermany, M. Goldbaum, W. Cai et al., "Identifying medical diagnoses and treatable diseases by image-based deep learning," Cell, vol. 172, no. 5, pp. 1122-1131, 2018.

[19] R. K. Samala, H.-P. Chan, L. Hadjiiski, M. A. Helvie, J. Wei, and K. Cha, "Mass detection in digital breast tomosynthesis: deep convolutional neural network with transfer learning from mammography," Medical Physics, vol. 43, no. 12, pp. 6654-6666, 2016. 
[20] G. Carneiro, J. Nascimento, and A. P. Bradley, "Unregistered multiview mammogram analysis with pre-trained deep learning models," in Medical Image Computing And Computer-Assisted Intervention, pp. 652-660, Springer International Publishing Ag, Cham, Switzerland, 2015.

[21] Y. Qiu, Y. Wang, S. Yan et al., "An initial investigation on developing a new method to predict shor-tterm breast cancer risk based on deep learning technology," in Medical Imaging 2016, Computer-Aided Diagnosis, San Diego, CA, USA, 2015.

[22] Z. Jiao, X. Gao, Y. Wang, and J. Li, "A deep feature based framework for breast masses classification," Neurocomputing, vol. 197, pp. 221-231, 2016.

[23] F. Gao, H. Yoon, T. Wu et al., "A feature transfer enabled multi-task deep learning model on medical imaging," Expert Systems with Applications, vol. 143, Article ID 112957, 2020.

[24] W. Sun, T.-L. Tseng, B. Zheng et al., "A Preliminary Study on Breast Cancer Risk Analysis Using Deep Neural Network," in Proceedings of the 13th International Workshop on Breast Imaging, IWDM 2016, pp. 385-391, Malmö, Sweden, June 2016.

[25] J. Arevalo, F. A. González, R. Ramos-Pollán, J. L. Oliveira, and M. A. Guevara Lopez, "Representation learning for mammography mass lesion classification with convolutional neural networks," Computer Methods and Programs in Biomedicine, vol. 127, pp. 248-257, 2016.

[26] Z. Wang, M. Li, H. Wang et al., "Breast cancer detection using extreme learning machine based on feature fusion with CNN deep features," Institute of Electrical and Electronics Engineers Access, vol. 7, pp. 105146-105158, 2019.

[27] Z. He, W. Lyu, G. Qin et al., "A feasibility study of building up deep learning classification model based on breast digital breast tomosynthesis image texture feature extraction of the simple mass lesions," Chinese Journal of Radiology, vol. 52, no. 9, pp. 668-672, 2018.

[28] T. Leung and J. Malik, "Representing and recognizing the visual appearance of materials using three-dimensional textons," International Journal of Computer Vision, vol. 43, no. 1, pp. 29-44, 2001.

[29] S. Lazebnik, C. Schmid, and J. Ponce, "A sparse texture representation using local affine regions," Institute of Electrical and Electronics Engineers Transactions on Pattern Analysis and Machine Intelligence, vol. 27, no. 8, pp. 12651278, 2005.

[30] U. R. Acharya, W. L. Ng, K. Rahmat et al., "Shear wave elastography for characterization of breast lesions: shearlet transform and local binary pattern histogram techniques," Computers in Biology and Medicine, vol. 91, pp. 13-20, 2017.

[31] C. Sanderson and K. K. Paliwal, "Identity verification using speech and face information," Digital Signal Processing, vol. 14, no. 5, pp. 449-480, 2004.

[32] A. J. Ma, P. C. Yuen, and J.-H. Jian-Huang Lai, "Linear dependency modeling for classifier fusion and feature combination," Institute of Electrical and Electronics Engineers Transactions on Pattern Analysis and Machine Intelligence, vol. 35, no. 5, pp. 1135-1148, 2013.

[33] D. C. Luvizon, H. Tabia, and D. Picard, "Learning features combination for human action recognition from skeleton sequences," Pattern Recognition Letters, vol. 99, pp. 13-20, 2017.

[34] C. Strobl, A.-L. Boulesteix, T. Kneib et al., "Conditional variable importance for random forests," Bmc Bioinformatics, vol. 9, 2008.

[35] D. M. Reif, A. A. Motsinger, B. A. McKinney et al., "Feature selection using a random forests classifier for the integrated analysis of multiple data types," in Proceedings of the 2006 Institute of Electrical and Electronics Engineers Symposium on Computational Intelligence in Bioinformatics and Computational Biology, p. 171, Institute of Electrical and Electronics Engineers, Kunming, China, April 2006.

[36] A. Verikas, A. Gelzinis, and M. Bacauskiene, "Mining data with random forests: a survey and results of new tests," Pattern Recognition, vol. 44, no. 2, pp. 330-349, 2011.

[37] S. Aydin, N. Arica, E. Ergul et al., "Classification of obsessive compulsive disorder by EEG complexity and hemispheric dependency measurements," International Journal of Neural Systems, vol. 25, no. 3, 2015.

[38] C. Cortes and V. Vapnik, "Support-vector networks," Machine Learning, vol. 20, no. 3, pp. 273-297, 1995.

[39] R. S. Lee, F. Gimenez, A. Hoogi et al., "A curated mammography data set for use in computer-aided detection and diagnosis research," Scientific Data, vol. 4, Article ID 170177, 2017.

[40] I. C. Moreira, I. Amaral, I. Domingues et al., "INbreast: Toward a Full-Field Digital Mammographic Database," Academic Radiology, vol. 19, no. 2, pp. 236-248, 2012.

[41] J. Suckling, J. Parker, D. Dance et al., "Mammographic image analysis society digital mammogram database exerpta medica," in Proceedings of the International Congress Series, vol. 1069, pp. 375-378, Kyoto, Japan, March 1994.

[42] S. M. Pizer, E. P. Amburn, J. D. Austin et al., "Adaptive histogram equalization and its variations," Computer Vision, Graphics, and Image Processing, vol. 39, no. 3, pp. 355-368, 1987.

[43] Q. Dai, "A competitive ensemble pruning approach based on cross-validation technique," Knowledge-Based Systems, vol. 37, pp. 394-414, 2013.

[44] M. Hussain, "Effective extraction of gabor features for false positive reduction and mass classification in mammography," Applied Mathematics \& Information Sciences, vol. 8, no. 1, pp. 397-412, 2014.

[45] L. Fangyi, S. Changjing, L. Ying et al., "Interpretable mammographic mass classification with fuzzy interpolative reasoning," Knowledge-Based Systems, vol. 191, Article ID 105279, 2019.

[46] N. Dhungel, G. Carneiro, and A. P. Bradley, “A deep learning approach for the analysis of masses in mammograms with minimal user intervention," Medical Image Analysis, vol. 37, pp. 114-128, 2017.

[47] C. Yuanqin, Z. Qian, W. Yaping et al., "Fine-tuning ResNet for breast cancer classification from mammography," in Proceedings of 2 nd International Conference on Healthcare Science and Engineering, pp. 83-96, Guilin, China, May 2019. 